Revista Electrónica Teoría de la Educación.

Educación y Cultura en la Sociedad de la Información.

http://www.usal.es/teoriaeducacion

Vol. 7. No2. Diciembre 2006

\title{
ALTERIDAD Y EMOCIONES EN LAS COMUNIDADES VIRTUALES DE APRENDIZAJE
}

El artículo trata de un conjunto de reflexiones sobre las relaciones interpersonales y las emociones en las comunidades virtuales de aprendizaje ${ }^{1}$. Los referentes teóricos se inspiran en los constructos bajtinianos de alteridad y dialogicidad y se complementan con las perspectivas de Vygotski sobre la construcción social del conocimiento y las emociones. Las relaciones interpersonales en las comunidades virtuales de aprendizaje y, sobretodo, las emociones vivenciadas en estos contextos son estudiadas en el ámbito del análisis de los discursos producidos en los foros de discusión. Los mensajes publicados y discutidos por los estudiantes y los profesores son analizados en el marco de la aplicación de metodologías cualitativas y del análisis de redes sociales. Los resultados preliminares de la investigación enfatizan la idea que, en las dos comunidades de virtuales de aprendizaje estudiadas, las relaciones interpersonales son reguladas por valores compartidos, por las emociones y los conflictos vivenciados. En este sentido, las emociones no han de estudiarse por el impacto que producen sobre el aprendizaje, sino en cuantos elementos integrados en el proceso de aprendizaje.

Palabras Clave: Alteridad, dialogicidad, comunidades virtuales de aprendizaje, relaciones interpersonales, emociones en línea.

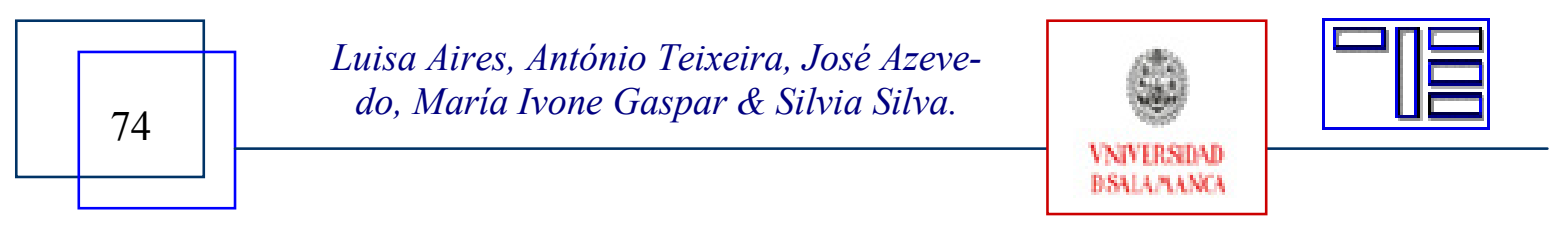




\section{ALTERITY AND EMOTIONS IN VIRTUAL LEARNING COMMUNITIES}

This paper reveals some ideas concerning interpersonal relations and emotions in virtual learning communities ${ }^{2}$. The main theoretical referents are founded in Bakhtin's constructs of alterity and dialogy and are enriched by Vygostsky's perspectives about social construction of knowledge and emotions. Interpersonal relations in virtual learning communities and, particularly, emotions experienced in these online contexts are studied through the analysis of discourses produced in discussion forums. These discourses that are published and discussed are analysed according to qualitative methodologies and reticular analysis of social interactions. The preliminary conclusions of this research show that, in the two virtual learning communities that were observed, the interpersonal relations are ruled not only by affinities and partnership but also by emotions and conflicts. These emotions should not be analysed according to their impact on learning; they should be looked as elements that make part of learning.

Key Words: Alterity, dialogy, virtual learning communities, interpersonal relations, emotions.

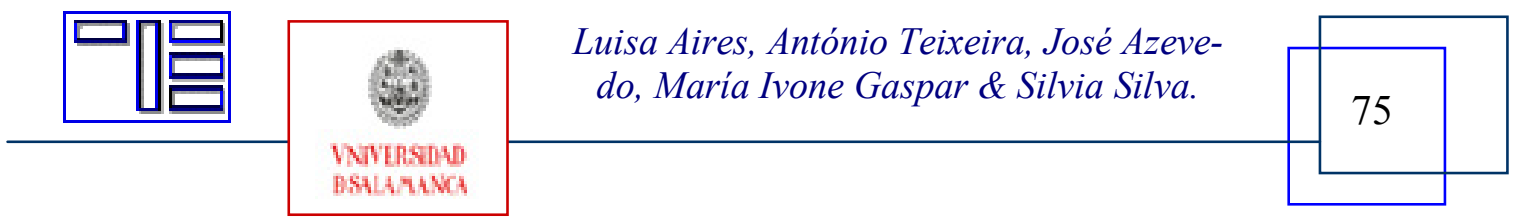


Revista Electrónica Teoría de la Educación.

Educación y Cultura en la Sociedad de la Información.

http://www.usal.es/teoriaeducacion

Vol. 7. No2. Diciembre 2006

\section{ALTÉRITÉ ET EMOTIONS DANS LES COMMUNAUTES VIRTUELLES D'APPRENTISSAGE}

Cet article présente quelques idées sur les relations interpersonnelles et les émotions dans les communautés virtuelles d'apprentissage ${ }^{3}$. Les référents théoriques principaux sont fondés à la théorie de Bakhtin. Les concepts d'altérité et de dialogie sont complémentés par la vision de Vygotsky sur la construction sociale de la connaissance et les émotions. Les relations interpersonnelles dans les communautés virtuelles d'apprentissage, en particulier, les émotions expérimentées dans ces contextes on-line sont étudiées parmi l'analyse des messages publiées aux forums de discussion. Ces discours qui sont édités et discutés par les étudiants et le professeur sont analysés dans les méthodologies qualitatives et de l'analyse réticulaire des interactions sociales. Les conclusions préliminaires de cette recherche éclairent que, dans les deux communautés virtuelles d'apprentissage, les relations interpersonnelles ne sont régulées seulement par les affinités mais également par les émotions et les conflits. Ces émotions ne devraient pas être analysées dans leur action sur l'apprentissage, mais également regardées comme éléments qui intègrent ce même apprentissage.

Mots clés: Altérité, dialogie, communautés virtuelles d'apprentissage, relations interpersonnelles, émotions on-line.

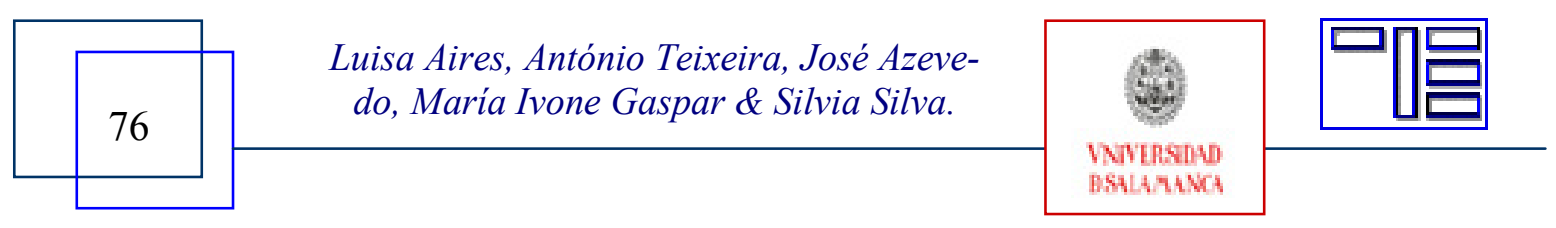




\title{
ALTERIDAD Y EMOCIONES EN LAS COMUNIDADES VIRTUALES DE APRENDIZAJE
}

\author{
Luísa Aires ${ }^{1}$, António Teixeira ${ }^{1}$, José Azecedo ${ }^{2}$, María Ivone Gaspar ${ }^{1}$ y Sílvia Silva ${ }^{1}$. \\ laires@univ-ab.pt; amt@univ-ab.pt; azevedo@letras.up.pt; migaspar@univ-ab.pt; \\ silvia@univ-ab.pt \\ Universidade Aberta [1], Universidade do Porto [2]
}

\section{1.- INTRODUCCIÓN}

Las problemáticas de la alteridad, polifonía, diversidad discursiva y construcción social de conocimiento, privilegiadas en el pensamiento educativo actual, son retos importantes en la identificación de nuevos caminos educativos que los principios educacionales modernos, asociados al dominio de la razón y la verdad única, no han sido capaces de precisar (Rebollo, 2001). La Sociedad Red (Castells, 1996) y su consecuente necesidad de reinventar de la vida cotidiana, no coexiste pacíficamente con esquemas de acción educativa de raíz apriorística, estática, lineal, sino que propone esquemas de acción centrados en la resolución de problemas no-estructurados, típicamente asociados a los sistemas sociales humanos. Como afirma Checkland (De Pablos, 1998:60 ) "un problema relacionado con las manifestaciones del mundo real de los sistemas de actividad humana es una condición caracterizada por un sentido de desajuste, que elude la definición precisa, entre lo que se percibe como la realidad y lo que se percibe que podría ser la realidad" (1993:180).

Intentando explorar y comprender dimensiones específicas de los fenómenos multidimensionales del aprendizaje en línea, en ámbito universitario, en este articulo pretendemos, en primer lugar, contribuir a la identificación de las dinámicas de interacción en las comunidades virtuales de aprendizaje, considerandose la comunicación dialógica y las emociones importantes dimensiones de los procesos de construcción identitaria de las Personas Adultas y, en segundo lugar, reflexionar sobre las potencialidades de las metodologías interpretativas del discurso, en el estudio de las interacciones en las comunidades de aprendizaje.

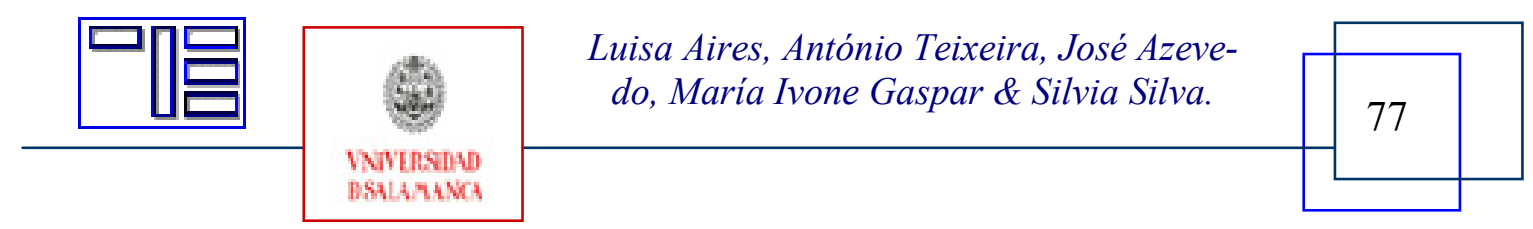


Revista Electrónica Teoría de la Educación.

Educación y Cultura en la Sociedad de la Información.

http://www.usal.es/teoriaeducacion

Vol. 7. No2. Diciembre 2006

\section{2.- ALTERIDAD, DIÁLOGO Y CONOCIMIENTO COMPARTIDO}

En la teoría sociocultural, la reflexión sobre la acción educativa exige la integración en el debate de los constructos de alteridad, dialogo y construcción social del conocimiento. Se considera el aprendizaje como un proceso básicamente interpersonal.

La construcción del self, el desarrollo de la "mismidad" (selfhood), por utilizar la expresión de Carl Rogers en su obra On Becoming a Person (1961), es básicamente un proyecto que encuentra su sentido en el confronto dinámico con la alteridad, en la circunscripción del mundo. En la alteridad, constructo que atribuye al otro un rol activo en el proceso de la comunicación, se genera la conciencia: "nuestro pensamiento se origina y se forma en el proceso de interacción con pensamientos ajenos" (Bakhtin, 1995, 93). En la acción pedagógica, "el ego no es el 'mío propio', sino 'el del otro', de ahí la definición de la acción pedagógica como acción social de la alteridad” (Mèlich, 1994, 111). Mèlich reflexiona en torno al anthropos como ser creador. Esta creación tiene lugar en el contexto de la reciprocidad del yo con el otro y en las relaciones sociohistóricas que coexisten en la vida cotidiana. Los escenarios educativos en línea no son escenarios vacíos, tabulas rasas para el individuo, sino redes de compromisos con múltiples otros que dibujan el itinerario personal del individuo que aprende. En este sentido, los ambientes de aprendizaje en línea ofrecen al yo un campo de desdoblamiento del sí-mismo en otro si discursivo, que se desglosa del primero de un modo narrativo, como si realmente del otro se tratara. Como sugiere Paul Ricoeur en su obra Soi-même comme un autre (1990), del confronto narrativo del yo con el sí-mismo (distanciación autobiográfica), se construye permanentemente el self. Es en esta relación de alteridad que se va edificando la acción educativa, en cuanto acción social mediada por múltiplas voces.

La apología de la naturaleza discursiva y dialógica de la experiencia ha sido tema de frecuentes reflexiones en el circulo de Bajtín (1992). Este grupo de intelectuales considera que la experiencia se crea a través del discurso y consecuentemente no tiene existencia fuera del campo de la expresión. En el pensamiento bajtiniano, el dialogo se entiende como una forma primordial del lenguaje. En este sentido, el intercambio comunicativo entre los agentes educativos emerge como actividad humana mediada por los escenarios, intencionalidades e instrumentos.

En la teoría sociocultural, la investigación sobre el dialogo en la acción educativa nos permite estudiar los procesos de la enseñanza y del aprendizaje en una perspectiva de construcción conjunta del conocimiento (Rogoff, 1994). Enseñanza y aprendizaje son integradas en un proceso dialógico continuo, de creación conjunta de significados orientados hacia la construcción de una conciencia crítica (Freire, 1968; Giroux, 1997), de un discurso propio (Bakhtin, 1995).

La perspectiva dialógica bajtiniana que adoptamos insiste en la idea de que yo puedo comunicar lo que yo sé, pero indirectamente, a través de palabras que me ofrece la comunidad y que restituyo a esta misma comunidad. Mi voz puede significar solamente en

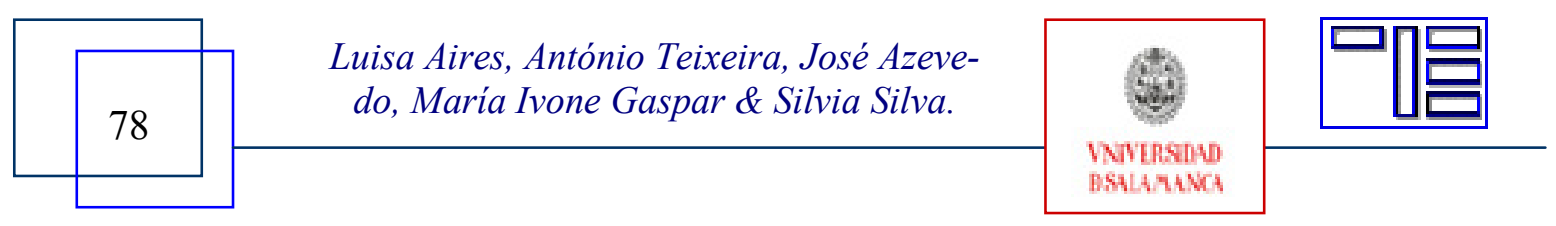


conjunto con otras voces (Wertsch, 1993) o como afirma Barresi “(...) intentamos comprender nuestra perspectiva, a partir de la perspectiva de una tercera persona e intentamos comprender el otro, a partir de una perspectiva personal" (Barresi, 2002).

El análisis de las relaciones interpersonales enmarcada en el pensamiento de Bajtin, nos permite reflexionar sobre los diálogos e interacciones entre agentes educativos, privilegiando los modos como construimos y compartimos el pensamiento (Aires, 2000), desarrollamos los valores, construimos universos y alianzas intelectuales, promovemos la integración del actor en el self, desarrollamos y consolidamos la construcción de identidades (Gonçalves, 2004). En última instancia, el estudio de los procesos de apropiación del conocimiento centrado en los discursos y en los escenarios de la acción educativa contribuye a la profundización del conocimiento sobre el desarrollo humano.

\section{3.- UNA PERSPECTIVA DIALÓGICA DE LAS EMOCIONES}

A pesar de marginado durante un largo periodo de tiempo por el pensamiento moderno (Damásio, 1996), el tema de las emociones ha sido objeto de estudio en investigaciones diversas, de raíz filosófica, psicológica, social, biológica, en la ultima década ${ }^{4}$. Las investigaciones desarrolladas en el ámbito de la teoría sociocultural (Gover y Gavelek, 1996), si bien atribuyen a las emociones el estatuto de fenómenos valorados en la esfera privada, consideran que estos fenómenos son definidos y aprendidos en las esferas publicas y sociales y son interpretados en cuanto representaciones internas de las reglas sociales. Gover y Gavelek (1996), en la perspectiva sociocultural de Vygotski, consideran que el conocimiento se integra en un sistema dinámico fundado en las dimensiones afectivas e intelectuales de la experiencia humana.

La relación de complementariedad entre las perspectivas de Vygotski y Bajtin favorece un planteamiento dialógico de las emociones, en cuanto proceso interpersonal mediado por voces que se crean y se desarrollan en dinámicas múltiples de reciprocidad permanente. Las emociones son elementos de las narrativas sociales e individuales y son estudiadas como instrumentos mediadores orientados para el self. Este planteamiento privilegia el estudio comunicacional de las emociones, entendiéndose la comunicación emocional como un espacio dinámico de construcción de nuevos significados y acciones. En este enfoque de las emociones, se privilegian los actos discursivos orientados hacia los usos del lenguaje en situaciones particulares (Bamberg, 1997). Los procesos afectivos se analizan en su dimensión narrativa, en los vínculos que el individuo establece con otras personas, lugares y acontecimientos, convirtiéndose en personas significativas, modelos de referencia y recursos culturales para su identidad (Rebollo, 2005). Los vínculos afectivos que creamos con el mundo fundan nuestras acciones sociales. De estas acciones y vínculos, privilegiamos los que se observan en las comunidades virtuales de aprendizaje.

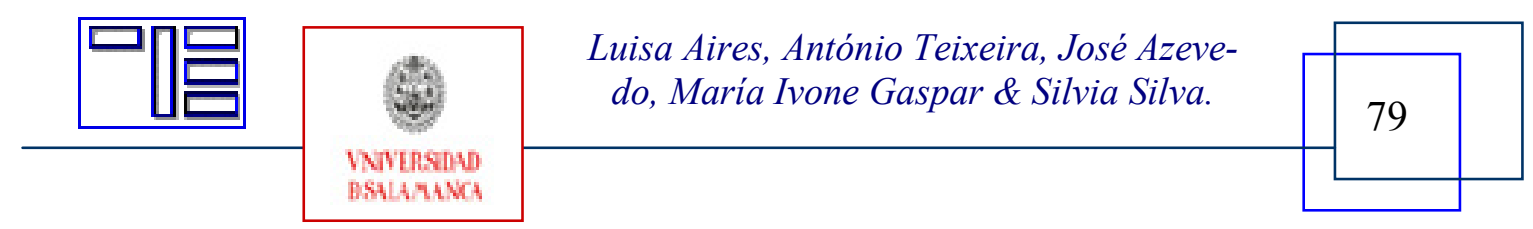


Revista Electrónica Teoría de la Educación.

Educación y Cultura en la Sociedad de la Información.

http://www.usal.es/teoriaeducacion

Vol. 7. No2. Diciembre 2006

\section{4.- COMUNIDADES DE VIRTUALES DE APRENDIZAJE Y COMUNICA- CIÓN EMOCIONAL}

Internet constituye una oportunidad única para la expresión de la multiplicidad del self (Turkle, 1995; Etchevers, 2005). Turkle y Etchevers cuestionan las tesis que asocian el implemento de las relaciones en línea a la tendencia para volvernos progresivamente seres asociales, aislados, solitarios y depresivos. En el intento de aclarar este planteamiento, proponemos la identificación de respuestas a las siguientes cuestiones: ¿Las relaciones en las comunidades virtuales son "frías" e "impersonales"? ¿Cómo se perciben las emociones en los escenarios educativos en línea?

En la búsqueda de sentidos y de respuestas a estas cuestiones, hemos estudiado las comunidades virtuales de aprendizaje, explorando aspectos inherentes a las relaciones interpersonales que se promueven en estas comunidades. Consideramos, siguiendo a autores como Vygostski, Bodor, Gavelek e Gover, que la evaluación de las situaciones y de las respuestas a estas situaciones son aprendidas en los contextos sociales. En este sentido, las emociones no han de ser analizadas en función del impacto que tienen sobre el aprendizaje, sino en cuanto dimensiones esenciales de este mismo aprendizaje: "La calidad emergente de la emoción tiene implicaciones importantes para la educación. (...) indica nuestra responsabilidad hacia la conscientización progresiva de que los procesos de enseñanza y de aprendizaje no son trasmisivos, sino relacionales" (Gavelek e Gover, 1996, 11).

\subsection{Comunidades de Aprendizaje}

El 'fenómeno individualizado' y la 'cultura particularizada' coexisten y se confrontan progresivamente con el 'fenómeno socializado' y la 'multiculturalidad. Ante esta coexistencia y este confronto se proponen argumentos, sujetándoles a la necesaria adecuación. El aprendizaje, en la sociedad de la información, no ha de limitarse únicamente a lo que es percibido en el ambiente único del aula pre-diseñada. Cada vez más, son relevantes otros ambientes que proponen otras estrategias de acción orientadas hacia resultados que valoran el desarrollo de competencias ajustadas a las nuevas exigencias personales y sociales.

En el año de 1989, Levin e Rumberger (citados por Saso et al., 2005) han comprobado que los cambios producidos en los procesos de trabajo generan nuevas competencias que a su vez se explicitan en las capacidades de iniciativa, cooperación, trabajo en grupo, formación mutua, evaluación, comunicación, resolución de problemas, tomada de decisiones, utilización de la información y planificación. La sociedad ha cambiado y en la Educación se impuso la necesidad de implicar, en esta trasformación, a todos los agentes. Una de las respuestas a esta necesidad se encuentra en la aplicación del sentido comunitario asociado al aprendizaje, en el proceso de participación y colaboración con y por el diálogo que ha de proporcionar la construcción de las comunidades de aprendi-

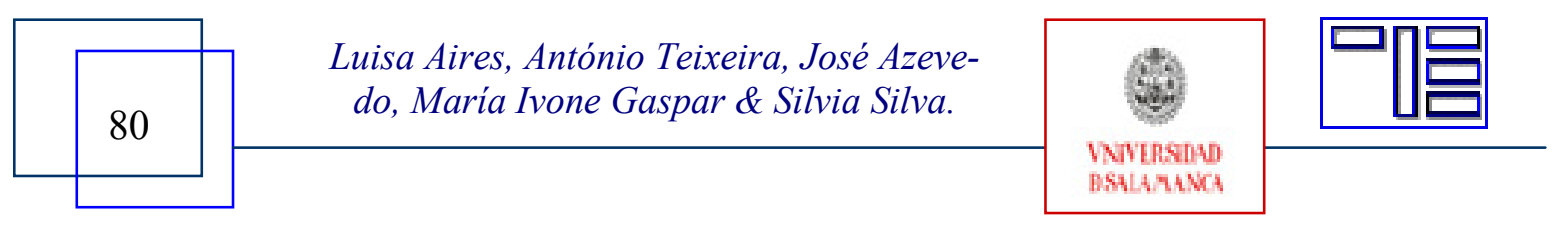


zaje. Se espera que las comunidades desarrollen procesos colaborativos de conocimiento.

Dias y Silva (2005) afirman que el enfoque de la colaboración centrado en los conceptos de polifonía, interactividad y dialogismo en los ambientes virtuales de aprendizaje, supone un importante reto a la educación a distancia. La interactividad promueve vínculos de aprendizaje suportados por el diálogo. Los autores destacan sobre este tema, cuatro elementos significativos: a) la manifestación natural de las intencionalidades colectivas; b) las reflexiones contextualizadas consecuentes a los cambios de experiencias, a través de los diálogos; c) el juego de alteridad y el placer en compartir conocimiento; d) la cooperación orientada hacia la colaboración.

Las comunidades virtuales de aprendizaje, núcleos privilegiados de la acción educativa en línea son constituidas por grupos de individuos, conectados a la red que comparten objetivos y promueven el ejercicio de la igualdad de derechos, de participación y de colaboración entre sus miembros (Palloff e Pratt, 1999). Podemos afirmar que la comunidad de aprendizaje se organiza a partir de tres constructos básicos e interdependientes: la dialogicidad, la polifonía y la interacción. En el cruce de la interacción y la dialogicidad, el aprendizaje acentúa modos comunes de explorar perspectivas controvertidas y de aprender atribuyendo sentido a los fenómenos complejos de la vida. De este modo, se acentúa el compromiso del estudiante ante el qué y el como aprender. El aprendizaje se perspectiva como un proceso que supone la participación social e individual; las acciones de compartir y de integrar fomentan la construcción de una comunidad. Si bien la creación de la comunidad puede justificarse por finalidades diversas, se identifican, igualmente, estrategias comunes: la participación horizontal, la comunicación dialógica y el compromiso recíproco.

En síntesis, es posible vincular a las comunidades de aprendizaje las siguientes ideas clave:

- El conocimiento es socialmente construido;

- La interacción se orienta hacia el aprendizaje individual; la adquisición de conocimiento resulta de la participación en las formas diversas de interacción y comunicación sociales;

- El lenguaje, artefacto social y cultural, es un instrumento mediador clave de la comunicación y de la interacción;

- Las actividades de pesquisa y de interpretación conjunta ayudan a los estudiantes a la adquisición de posturas activas y críticas en la construcción de su propia identidad;

- La tecnología tiene potencialidades para soportar formas diversas de interacción social, de comunicación y de colaboración, en las tareas de construcción de conocimiento;

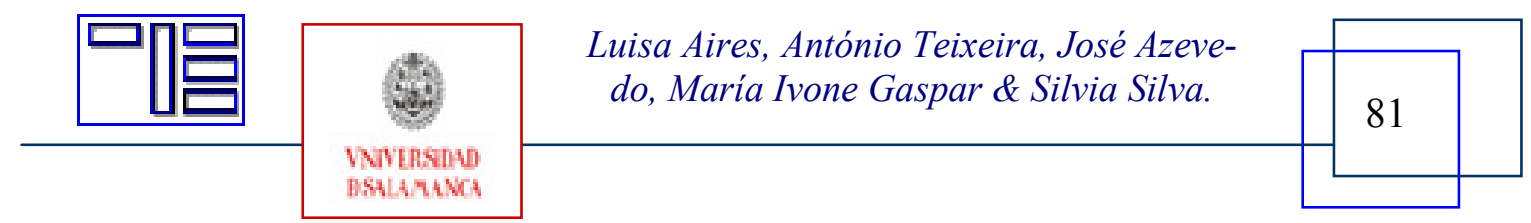


Revista Electrónica Teoría de la Educación.

Educación y Cultura en la Sociedad de la Información.

http://www.usal.es/teoriaeducacion

Vol. 7. No2. Diciembre 2006

- La autoridad y la regulación del conocimiento se trasladan hacia los alumnos gradualmente; se estimula el desarrollo de nuevos conocimientos, capacidades y el autoconcepto;

- El conocimiento se distribuye entre los miembros de la comunidad; los estudiantes son productores y utilizadores del conocimiento, a través de la colaboración y la comunicación entre iguales.

Estas ideas, una vez aplicadas a las comunidades virtuales de aprendizaje, nos permiten comprender estas comunidades, en cuanto núcleos integradores de las sociabilidades del ciberespacio. En dichos núcleos, los foros de discusión son "territorios" privilegiados para el estudio de estas dinámicas. En los espacios comunicativos de los foros coexisten niveles diferenciados de información, de estrategias de resolución de problemas y una diversidad de modos discursivos. El texto compartido, en los foros, es una especie de mediador de comunicación, restituyéndose a la intersubjectividad una posición teórica determinante para la interpretación del conocimiento compartido (Turoff, 1991).

Contrariamente a los chats, los foros de discusión suponen la creación de cadenas de mensajes, diferidas en el tiempo, que permiten a los utilizadores introducir nuevas temáticas, retomar y profundizar temáticas previamente publicadas, enriqueciéndose con nuevas perspectivas (Domínguez y Alonso, 2005). En estos foros se pueden identificar, entre otras dimensiones, relaciones entre la calidad y la cantidad de participación, el nivel de información de los mensajes publicados, las modalidades discursivas, los mecanismos de resolución de problemas.

En el estudio empírico que presentamos a continuación hemos aplicado, dominantemente, metodologías de raíz cualitativa. A partir del análisis de los mensajes publicados en los foros, accedemos a la naturaleza de las interacciones educativas en los espacios virtuales (Smolka, 1996; De Pablos et al., 1999; Rebollo, 2001). Posteriormente, el análisis cualitativo de los discursos ha sido complementado con el análisis de redes sociales, de raíz cuantitativa (Domínguez y Alonso, 2005).

\section{2- Dinámicas interpersonales en un foro de discusión: Caso A}

En este primer estudio de caso, pretendemos explorar modalidades y dinámicas de relación interpersonal en los foros de discusión y, de un modo particular, desarrollar una metodología de análisis de las dinámicas de interacción en línea, en ámbitos educativos. Para ello, hemos analizado los mensajes publicados en un foro de discusión asíncrono y discrepante ${ }^{5}$ que ha sido desarrollado en el marco de una asignatura de un curso de posgrado (maestría) en línea.

Esta comunidad de aprendizaje está constituida por 15 estudiantes ( 12 hombres y 3 mujeres) y el profesor. Los estudiantes que frecuentan este posgrado son maestros y profesores de diferentes niveles de enseñanza. De un modo general, el nivel de participación de esta comunidad, en los foros, es reducido a pesar de la raíz colaborativa del modelo

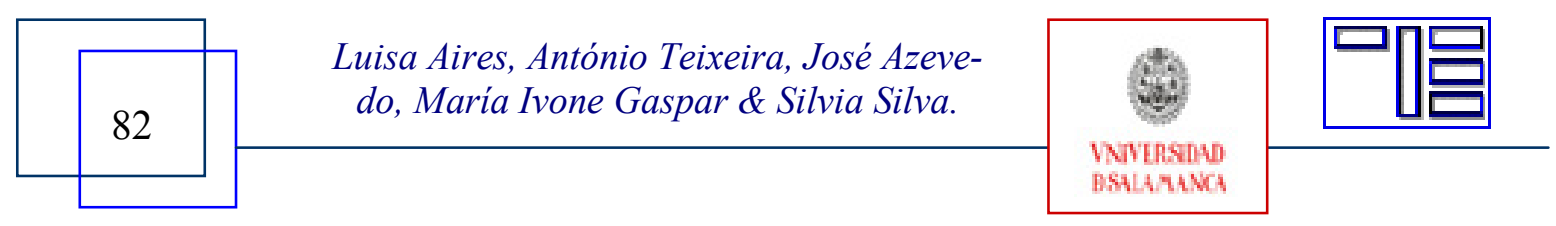


pedagógico que orienta el curso (Pereira et al, 2003). El foro estudiado ha sido desarrollado a lo largo de 16 días y durante este periodo se ha publicado un total de 100 mensajes.

El foro se analiza a partir de una unidad macro de análisis- el episodio (Vygotski,1986; Wertsch, 1993; De Pablos, 1995; Rebollo, 1999).En este foro, hemos identificado dos episodios con intencionalidades diferentes. El objetivo central del primer episodio se caracteriza por la presentación individual de las aportaciones de los estudiantes que surgen como respuesta a la actividad propuesta por el profesor.

El segundo episodio se caracteriza por la emergencia y desarrollo de una situación de conflicto, bajo el pretexto de profundización de la temática científica en estudio. Mientras el primer episodio presenta una secuencia típicamente escolar, con las respuestas de los estudiantes a la actividad solicitada por el profesor, el segundo episodio empieza con un mensaje que integra una aportación no solicitada ni esperada por el grupo y que, a su vez, es interpretada, por la mayoría de los estudiantes, como un intento de búsqueda de protagonismo ante el profesor. Esta situación ocasiona algunas actitudes límite de sorpresa y de protesta de algunos estudiantes. Ante estas actitudes, el estudiante, víctima del conflicto, contesta con mensajes que aparentan inquietud y perturbación. En esta espiral de emociones, se desarrolla una situación de conflicto grupal.

\subsection{1- Análisis reticular de las interacciones sociales}

Una vez explorados e interpretados los mensajes publicados en el foro, hemos analizado las interacciones sociales de esta comunidad virtual de aprendizaje, a través del análisis de redes sociales (Domínguez y Alonso, 2005). Esta metodología facilita la reflexión sobre los procesos sociales en el ámbito grupal (Hanneman e Riddle, 2005). Tomados como nodos en la red de comunicación, los mensajes nos permiten identificar la centralidad, densidad, intermediación y la proximidad de los agentes. Los datos que presentamos a continuación se refieren al grado de proximidad entre los miembros de la comunidad.

El análisis de las interacciones en los dos episodios identificados, nos ofrece un conjunto de dinámicas diferenciadas, en función de los objetivos comunicativos de la comunidad. El primer episodio (figura 1), observamos una red de interacciones con diferentes nodos, flujos multidireccionales y vínculos múltiples que sugieren una fuerte proximidad con el diagrama global y dominante de las interacciones en el foro. En esta red, la comunidad (DCSI) ocupa una posición central en las interacciones que traduce la intencionalidad de la comunidad en fomentar la cohesión del grupo (estudiantes: A01 hasta A15; profesor: P01).

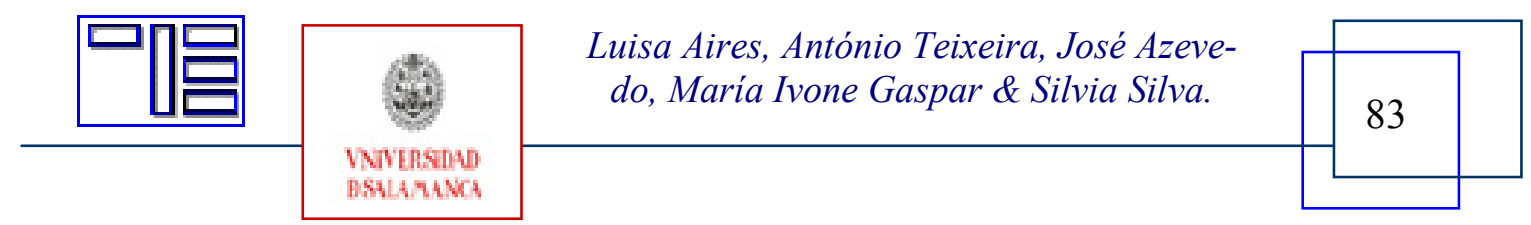


Revista Electrónica Teoría de la Educación.

Educación y Cultura en la Sociedad de la Información.

http://www.usal.es/teoriaeducacion

Vol. 7. No2. Diciembre 2006

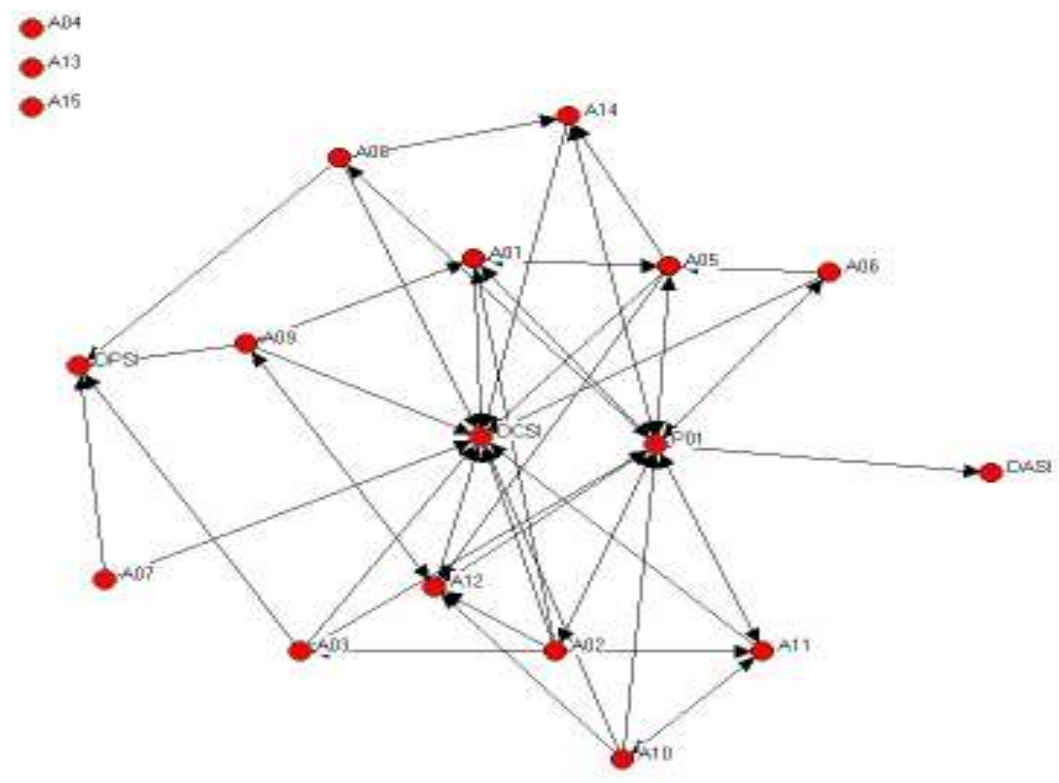

Figura 1: Representación de la Red de Interacciones en el Primer Episodio.

La red del segundo episodio (figura 2) representa una estructura diferente. La frecuencia de las interacciones entre algunos de los estudiantes se intensifica y culmina con actitudes de descalificación (Watzlawick, Bavelas y Jackson, 1967), bajo el pretexto de profundización de la discusión. La centralidad de la interacción se mueve hacia los estudiantes que promueven el conflicto y la comunidad parece dividirse en dos subgrupos: a favor y en contra la actitud de uno de los estudiantes.

La comunidad en su globalidad (DCSI) pierde la centralidad en las interacciones y se detecta una ruptura en la cohesión grupal y algunos de los estudiantes - A06, A07, A14, se alejan de la discusión. El estudiante A10 adquiere centralidad en la discusión, concluyéndose, a partir del análisis de sus mensajes, que este estudiante parece ignorar la necesidad del uso de un discurso socialmente deseable, en escenarios educativos. En el diagrama de la figura 2 observamos, también, que el profesor pierde la centralidad que ocupa en el episodio anterior.

En el análisis de los mensajes de este segundo episodio, se destaca el uso de estrategias de trolling que demuestran actitudes de ruptura:

"Cuando leemos el supuesto cuadro-síntesis, uno se queda con la idea que no has contestado a nadie. ¿Que has hecho tú en estos días de discusión? ¿No hubo nada, no hubo evolución en la construcción de tu conocimiento?" (A01)

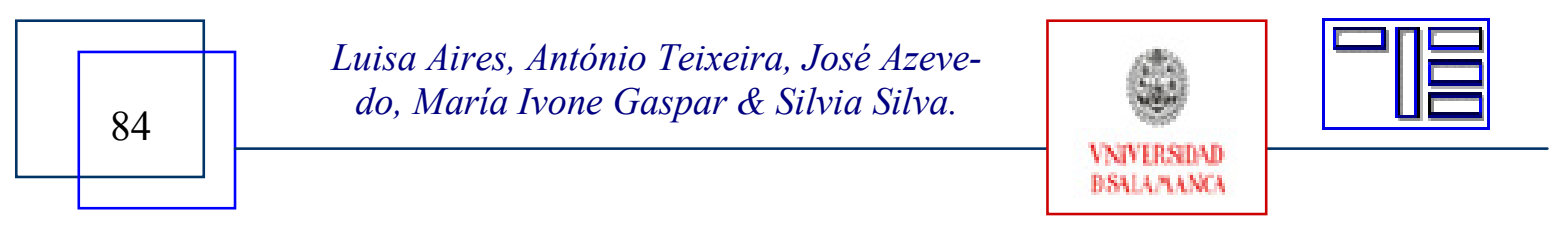



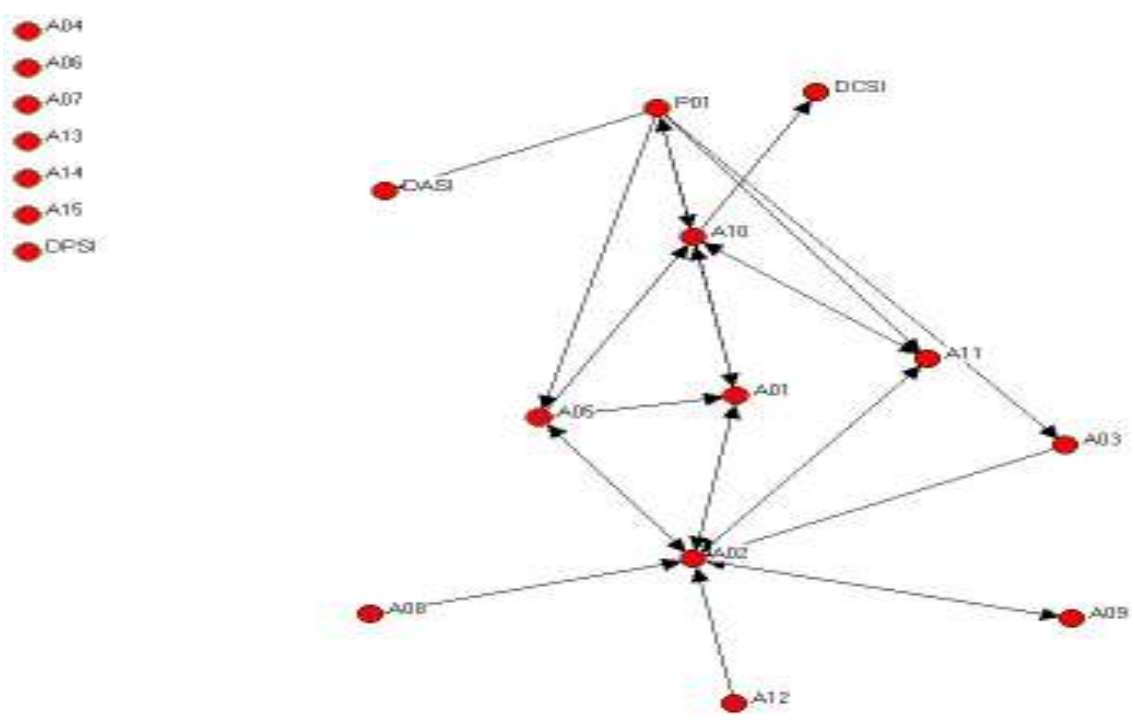

Figura 2: Representación Gráfica de la Red de Interacciones del Segundo Episodio.

Si complementamos el análisis de este último episodio con las reflexiones producidas sobre las experiencias emocionales a lo largo de un curso, por un segundo grupo de estudiantes de posgrado, se densifican y se profundizan las interpretaciones del estudio que hemos presentado en este apartado.

4.3.- Voces de los estudiantes sobre las emociones en escenarios educativos en línea: Caso B

El segundo caso nos aporta un conjunto de reflexiones producidas por un grupo de estudiantes, en un foro de discusión, sobre sus vivencias emocionales en línea. Este foro ha registrado una fuerte participación de un grupo de cinco estudiantes (mujeres) y un profesor. A este grupo se les ha planteado la siguiente pregunta en el foro de discusión: ¿Que roles tienen las experiencias emocionales en la comunidad virtual de aprendizaje?

Las respuestas a la pregunta han sido analizadas, privilegiándose las temáticas de los mensajes publicados. A partir de este análisis, se han identificado los núcleos temáticos que presentamos a continuación:

\section{- Foros de discusión:}

Los foros de discusión son percibidos por las estudiantes como contextos de aprendizaje colaborativo y en cuantos espacios donde se construyen emociones, sentimientos, solidaridades. Estas emociones son filtradas por la "mirada académica", se interpretan como experiencias "paralelas" subvaloradas por las actividades científicas y son frecuentemente externalizadas a través de los silencios.

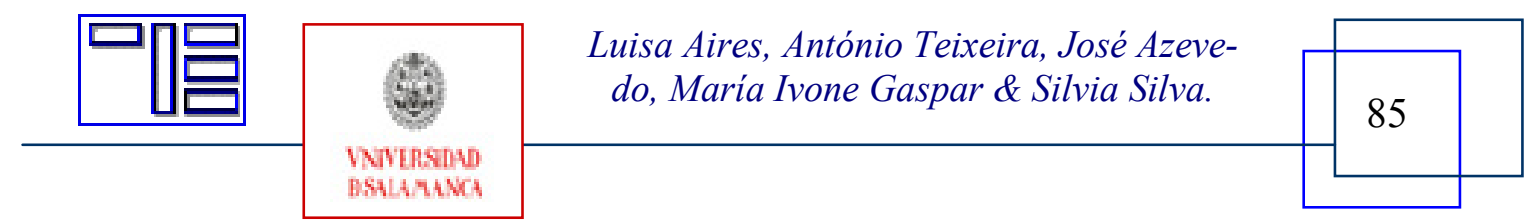


Revista Electrónica Teoría de la Educación.

Educación y Cultura en la Sociedad de la Información.

http://www.usal.es/teoriaeducacion

Vol. 7. No2. Diciembre 2006

\section{- Relaciones Interpersonales}

Esta comunidad de estudiantes considera que las relaciones interpersonales han de caracterizarse por la confianza reciproca y por la capacidad comprensión y de atención hacia el otro. El conflicto es un elemento intrínseco a las interacciones en línea y se diluye a través de la comunicación.

\section{- Lenguaje escrito}

La escritura es un instrumento básico para la expresión de las emociones en línea. El acto de escribir se entiende como un acto personal y el lenguaje escrito se interpreta como un instrumento facilitador y de expresión de emociones y sentimientos, un regulador de vínculos afectivos.

\section{- Emociones y sentimientos en línea}

Este grupo de estudiantes considera que las emociones son elementos esenciales de la condición humana que reflejan las experiencias de los individuos y poseen un rol fundamental en las comunidades virtuales de aprendizaje. Las emociones se descubren en las acciones de los agentes educativos, en sus percepciones sobre el otro y en los escenarios de aprendizaje, así como en los tipos de lenguaje escrito, las dificultades de los estudiantes, los recursos disponibles y en los mensajes de apoyo reciproco.

\section{5.- CONCLUSIONES}

Los dos casos que hemos presentado en este artículo se enmarcan en una investigación de raíz exploratoria e interpretativa y, por lo tanto, las conclusiones no son generalizables. Las dos comunidades virtuales de aprendizaje que hemos estudiado se sostienen a partir de dinámicas de aprendizaje dialogicas y son percibidas como espacios polifónicos, diversos y de construcción conjunta de experiencias. El sentido de comunidad se mantiene en el ámbito de los contextos comunicativos enraizados en la alteridad (Bakhtin, 1982; Wertsch, 1993).

Los foros de discusión, escenarios que promueven la presentación, la discusión y la profundización del conocimiento, proporcionan, también, la definición y negociación de valores y pautas de organización de la comunidad (Swan, 2002). A través del lenguaje escrito, instrumento mediador por excelencia en estos contextos de aprendizaje, se comparten conocimientos, sentimientos y actitudes y se contribuye a la cohesión de la comunidad.

En los dos cursos de posgrado en línea que hemos estudiado, los roles de los profesores $\mathrm{y}$ de los estudiantes cambian, relativamente a la enseñanza presencial tradicional. El profesor puede disfrutar de una postura central en la fase inicial de la actividad educativa, pero comparte, gradualmente, el poder y la centralidad en el aula, con los estudian-

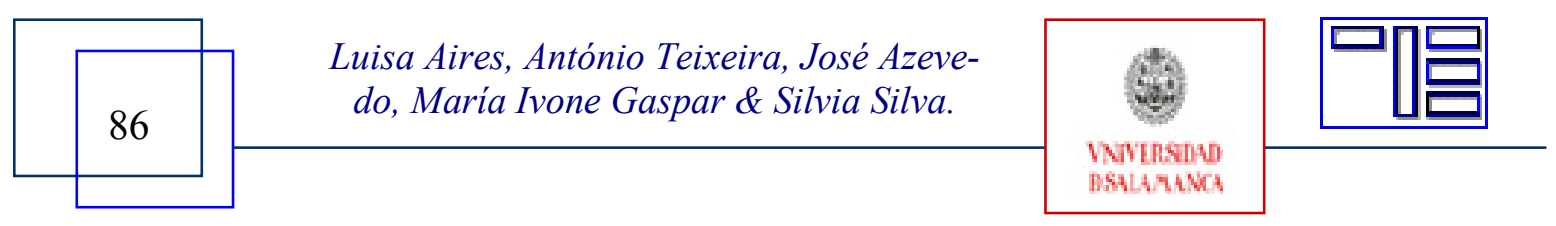


tes. Su rol dominante consiste, ahora, en moderar experiencias, aprendizajes y relaciones interpersonales. Esta nuevas prácticas son reveladoras de los cambios e innovaciones que la creación del Espacio Europeo de Educación Superior privilegia.

Los conflictos se entienden como elementos intrínsecos y de regulación de las comunidades virtuales de aprendizaje y pueden ser importantes delimitadores de las normas de funcionamiento y de las culturas grupales. Las emociones, a pesar de su frecuente estatuto subalterno, en las prioridades educativas, son importantes instrumentos mediadores en los aprendizajes. En esta investigación se corrobora la perspectiva de Swan (2002) sobre el papel determinante de las emociones en las comunidades virtuales de aprendizaje, como vía de compensación de la ausencia de la comunicación no-verbal, en los espacios de aprendizaje en línea. Los casos que hemos estudiado nos indican la urgencia en promover la socialización emocional, en ámbitos educativos $\mathrm{y}$, particularmente, en los escenarios de aprendizaje en línea.

Relativamente a las metodologías adoptadas en la investigación, se concluye que el análisis sociocultural de los discursos es una vía adecuada que facilita el acceso a la naturaleza de los discursos científicos y, sobretodo, a la multiplicidad de modalidades del discurso educativo. El análisis cuantitativo de redes sociales ha aportado una lectura complementar y enriquecedora de los discursos que hemos estudiado.

Por fin, hay que restituir la palabra a los actores principales de esta investigación y, por eso, ventriloquizamos la voz de una estudiante que comparte sus vivencias en su comunidad virtual de aprendizaje:

"Quizás, no volveré a encontrarme a los compañeros, profesores y los amigos que he conocido en este curso. Pero si sé, que si los encuentro, un día, en el mundo "virtual" y/o "real", desprenderé una lagrima de contentamiento, y estoy segura que será un placer recordar los momentos felices, tristes y difíciles que hemos compartido este curso.

[...] El final, en sí mismo, no existe. Los momentos siempre son eternos en nuestra memoria y el futuro nos reserva muchas sorpresas!" (PG.L)

\section{6.- BIBLIOGRAFÍA}

AIRES, L.(2000): Vozes sobre a televisão no âmbito da Educação de Pessoas Adultas: Uma abordagem sociocultural. Lisboa: Universidade Aberta (tesis de doctorado inédita) @PRENDE.COM - Comunidades, Aprendizagem e Comunicação Online. Disponible en: http://www.aprende.com.pt

BAKHTIN, M. (1982): Estética de la creacción verbal. México, Siglo XXI.

BAMBERG, M. (1997): Emotion talk(s): The role of perspective in the construction of emotions, in NIEMEIR, S. and DIRVEN, R. (eds.) The language of emotions: concep-

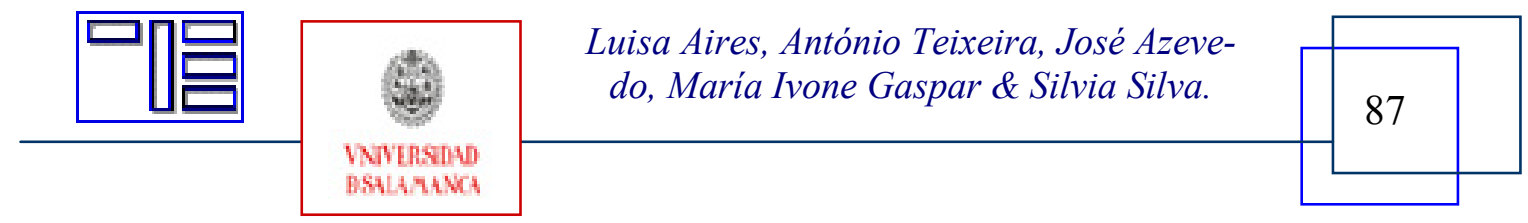


Revista Electrónica Teoría de la Educación.

Educación y Cultura en la Sociedad de la Información.

http://www.usal.es/teoriaeducacion

Vol. 7. No2. Diciembre 2006

tualization, expression and theoretical foundation. Amsterdam, John Benjamins B. V., 209-225.

BARRESI, J. (2002): From "the thought is the thinker" to "the voice is the speaker". William James and the Dialogical Self. . Disponible en:

http://jbarresi.psychology.dal.ca/Papers/Dialogical Self.htm

BELTRÁN, A. and ALONSO, J. (2005): "Critical Communicative Methodology". First International Congress of Quality Inquiry. University of Illinois, Urbana-Champaign" May, 5-7. Disponible en: http://www.c4g.org/gi2005/papers/beltran.pdf

CASTELLS, M. (1996): La Era de la Información. Economía, Sociedad y Cultura. (vol. 1). Madrid, Alianza Editorial

CHECKLAND, P. (1993): Pensamiento de sistemas, práctica de sistemas. México, Grupo Noriega Editores.

COLÁS, P. (2002): El análisis cualitativo de datos, in Buendía, L.; Colás, P. y Hernández, F. Métodos de Investigación en Psicopedagogía. Madrid, McGraw-Hill, 225-249.

DAMÁSIO, A. (1996), O erro de Descartes (16 ${ }^{\mathrm{a}}$ ed.). Lisboa, Publicações EuropaAmérica.

DE PABLOS, J. (1995): La mediación de la televisión en el ámbito de la educación de adultos (Diseño con un nivel microgenético). Sevilla, Facultad de Ciencias de la Educación (investigación inédita).

DE PABLOS, J. (1998): Nuevas tecnologías aplicadas a la educación: una vía para la innovación, in DE PABLOS, J. y SEGURA, J. (coords.) Nuevas Tecnologías, Comunicación Audiovisual y Educación. Barcelona, Cedecs Psicopedagogía, 49-70.

DE PABLOS, J., REBOLLO, M.A, AIRES, L.(1999): Para un estudio de las aportaciones de Mijail Bajtín a la teoría sociocultural. Una aproximación educativa, Revista de Educación, 223-253.Madrid: Ministerio de Educación

DIAS, S. e SILVA, M. (2005). Dialógica e Interactividade em Educação Online. In Revista FAEEBA - Educação e Contemporaneidade, Salvador, v. 14, n. ${ }^{\circ}$ 23, 169-179, Jan./Junho.

DOMÍNGUEZ, D. y ALONSO, L. (2005): Evaluación mixta de comunidades de aprendizaje en línea. Disponible en el ARCHIVO del Observatorio para la CiberSociedad en http://www.cibersociedad.net/archivo/articulo.php?art=205

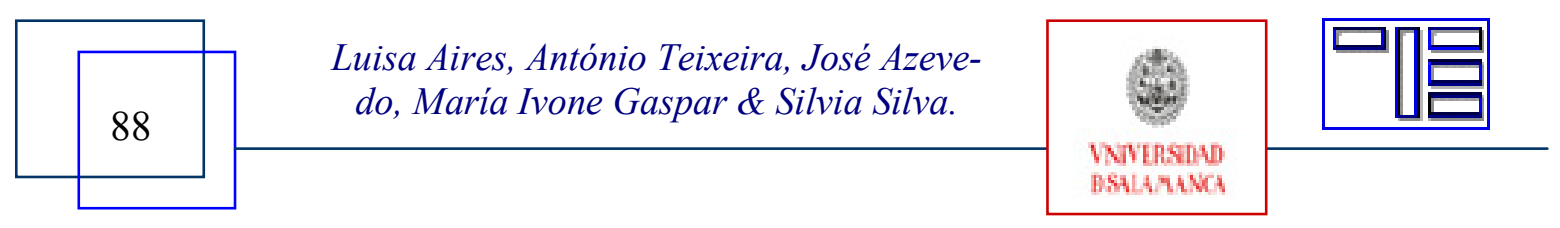


ETCHEVERS, N. (2005): ¿Dónde están las emociones en el Ciberespacio? Análisis de la situación actual, Revista TEXTOS de la CiberSociedad, 5. Temática Variada. Disponible en http://www.cibersociedad.net (consultado en 15.03.06).

FREIRE, P. (1968): Pedagogia do oprimido. Rio de Janeiro: Paz e Terra.

GAVELEK, J. and GOVER, M. (1996): Educating the emotions: implications of a relational view of knowing for learning and development. Disponible en: http://msu.edu/user/govermar

GIROUX, H. (1997): Cruzando límites. Trabajadores culturales y politicas educativas. Barcelona, Paidós.

GONÇALVES, M. (2004): "Identidade e narrativa pessoal", in TEIXEIRA, F. (coord.) Identidade Pessoal: Caminhos e Perspectivas. Coimbra, Quarteto, 165-179.

HANNEMAN, R. and RIDDLE, M. (2005): Introduction to social network methods. Riverside, University of California, Riverside. Disponible en: http://faculty.ucr.edu/ hanneman/

HARRÉ, R. (ed.) (1986): The social construction of emotions. New York: Basil Blackwell.

MÈLICH, J. (1994): Del extraño al cómplice: La educación en la vida cotidiana. Barcelona, Anthoropos.

PALLOFF, R.; PRATT, K. (1999): Building learning communities in cyberspace: effective strategies for the online classroom. San Francisco, Jossey-Bass Publishers.

PEREIRA, A.; QUINTAS, A.; MOTA, J.C.; MORGADO, L.; AIRES, L. (2003). "Contributos para uma pedagogia do ensino pós-graduado: proposta de um modelo". Revista Discursos, Série "Perspectivas em Educação", Novos Rumos e Pedagogia em Ensino a Distância, 39-51. Lisboa: Universidade Aberta

REBOLLO, M. (1999): La teoría sociocultural aplicada al estudio de la televisión en el ámbito de la educación de personas adultas. Tesis de doctorado, Departamento de Didáctica y Organización Escolar, Universidad de Sevilla (investigación inédita).

REBOLLO, M. (2001): Discurso y Educación. Sevilla, Mergablum.

REBOLLO, M. (2005): Emociones, Género y Identidad: La educación sentimental. Sevilla, Universidad de Sevilla (texto policopiado).

RICOEUR, P. (1990). Soi-même comme un autre, Paris: Le Seuil.

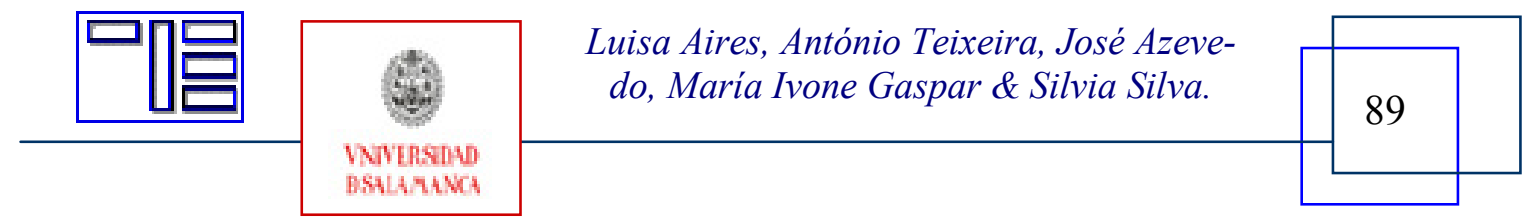


Revista Electrónica Teoría de la Educación.

Educación y Cultura en la Sociedad de la Información.

http://www.usal.es/teoriaeducacion

Vol. 7. No2. Diciembre 2006

RICOEUR, P. (1992 [1990]). Oneself as Another (Soi-même comme un autre), trans. Kathleen Blamey. Chicago: University of Chicago Press.

ROGERS, C. (1961). On becoming a person: A therapist's view of psychotherapy. London: Constable.

ROGOFF, B. (1994): Los aprendices del pensamiento. El desarrollo cognitivo en el contexto social. Barcelona, Paidós.

SASO, C. et al. (2005): Comunidades de Aprendizaje: transformar la educación. Barcelona, Editorial GRAÓ.

SMOLKA, A. (1996): Voices of discourse: some conceptions in issues in psychological studies, in II Conference of Sociocultural Research, Ginebra (documento policopiado).

SWAN, K. (2002): Building learning communities in online courses: the importance of interaction, Education, Communications and Information, 2 (1), 23-49.

TURKLE, S. (1995 ou 1997): La vida en la pantalla. La construcción de la identidad en la era de Internet. Barcelona, Paidós Ibérica.

TUROFF, M. (1991): Computer-Mediated Communication Requirements for Group Support (.pdf). Originally published in Journal of Organizational Computing, 1, 85113. Disponible en: http://web.njit.edu/ turoff/Papers/CMCGS.pdf

VOLOSHINOV, V./BAKHTIN, M. (1930, 1992): Marxismo e Filosofia da Linguagem. São Paulo: HUCITEC

VYGOTSKI, L. (1986): Mind in society. The development of higher psychological processes. Harvard Mass, Harvard University Press.

VYGOTSKI, L. (2004): Teoría de las emociones. Estudio histórico-psicológico. Madrid: AKAL.

WATZLAWICK, P; BAVELAS, J. and JACKSON, D. (1967): Pragmatics of human communication: A study of interactional patterns, pathologies and paradoxes. New York, Norton.

WERTSCH, J. (1993): Voces de la mente. Un enfoque sociocultural para el estudio de la acción mediada. Madrid, Visor.

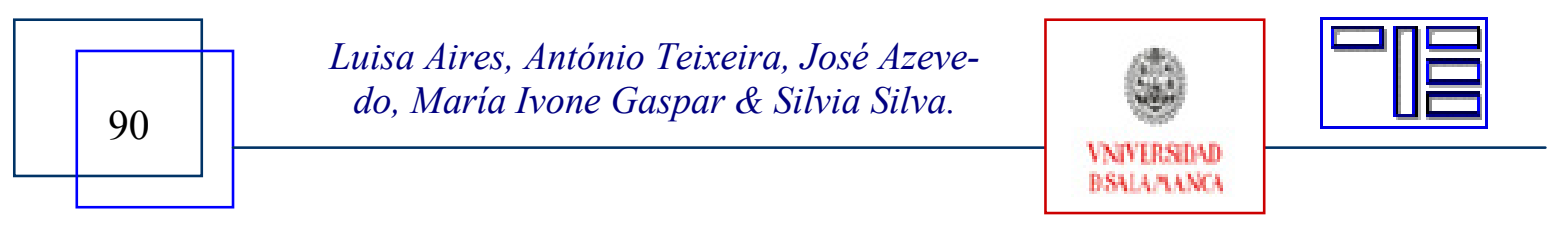




\section{NOTAS}

[1] Este estudio se integra en una investigación más amplia que actualmente estamos desarrollando en el ámbito del proyecto “@prende.com - Comunidades, Aprendizagem e Comunicação Online" - POCI/CED/59736/2004, subvencionado por la "Fundação para a Ciência e a Tecnologia". Uno de los objetivos más importantes del proyecto consiste en estudiar las dinámicas de apropiación de conocimiento, privilegiándose las relaciones sociales, en cursos de posgrado en línea y presenciales. Algunas de las actividades del proyecto pueden consultarse en: http://www.aprende.com.pt

[2] This study is a part of a bigger research - Project @learn.com - Communities, Learning and online Communication", supported by "Fundação para a Ciência e a Tecnologia". One of the main goals of this project is to study the dynamics of appropriation of knowledge and the relevance of social relations in online and face-to-face post-graduation courses. @prende.com is available at: http://www.aprende.com.pt

[3] Cette étude est une partie d'une recherche - Projet “@prende.com - Communautés, Apprentissage et Communication on-line", es soutenue par la "Fundação para a Ciência e a Tecnologia". Un objectif principal de ce projet est orienté a l'étude des dynamiques d'appropriation de la connaissance et de l'importance des relations sociales on-line dans les masters universitaires. Le projet "@prende.com" est disponible au: http://www.aprende.com.pt.

[4] Muchos de los temas desarrollados actualmente son tratados en el estudio histórico-psicológico de Vygotski (1896-1934) sobre las emociones.

[5] Hemos elegido un foro de discusión discrepante, a partir de las orientaciones propuestas por Colás (2002) sobre las técnicas generadoras. Estas técnicas tienen como objetivo facilitar la emergencia de conceptos y teorías y contrastar hipótesis rivales.

\section{Para citar este artículo puede utilizar la siguiente referencia:}

AIRES, L.; TEIXEIRA, A.; AZEVEDO, J.; GASPAR, M. I. y SILVA, S. (2006): Alteridad y emociones en las comunidades virtuales de aprendizaje. En GARCÍA CARRASCO, Joaquín (Coord.) Estudio de los comportamientos emocionales en la red [monográfico en línea]. Revista electrónica Teoría de la Educación: Educación y Cultura en la sociedad de la información. Vol. 7, nº 2. Universidad de Salamanca. [Fecha de consulta: $\mathrm{dd} / \mathrm{mm} / \mathrm{aaaa}]$.

$<\mathrm{http}: / /$ www.usal.es/ teoriaeducacion/rev_numero_07_02/n7_02_diego_levis.pdf > ISSN 1138-9737

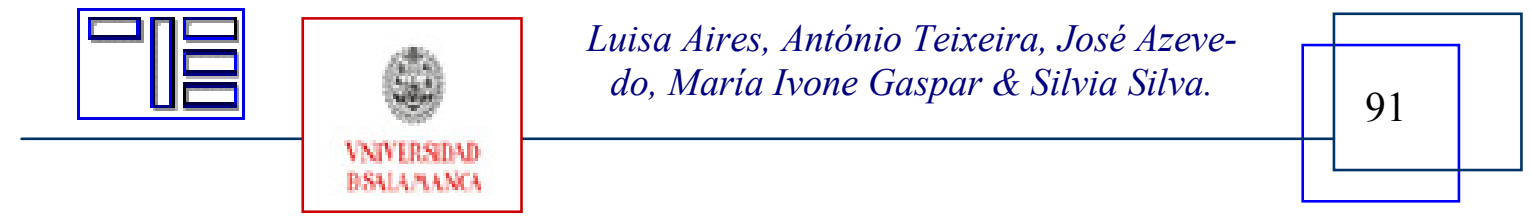

